\title{
Primitive prime divisors in the critical orbits of one-parameter families of rational polynomials
}

\author{
By RUFEI REN \\ Department of Mathematics, Fudan University, \\ 220 Handan Rd., Yangpu District, Shanghai 200433, China \\ e-mail: rufeir@fudan.edu.cn
}

(Received 27 October 2020)

\begin{abstract}
For a polynomial $f(x) \in \mathbb{Q}[x]$ and rational numbers $c, u$, we put $f_{c}(x):=f(x)+c$, and consider the Zsigmondy set $\mathcal{Z}\left(f_{c}, u\right)$ associated to the sequence $\left\{f_{c}^{n}(u)-u\right\}_{n \geqslant 1}$, see Definition 1.1], where $f_{c}^{n}$ is the $n$-st iteration of $f_{c}$. In this paper, we prove that if $u$ is a rational critical point of $f$, then there exists an $\mathbf{M}_{f}>0$ such that $\mathbf{M}_{f} \geqslant$ $\max _{c \in \mathbb{Q}}\left\{\# \mathcal{Z}\left(f_{c}, u\right)\right\}$.

\section{Contents}

1 Introduction

2 Introduction of Proposition $2 \cdot 2$ and some estimates

$2 \cdot 1 \quad$ Upper bounds for $\ln B_{n}$ and $\ln \left|g_{c}^{n}(c)\right|$

$2 \cdot 2$ A lower bound for $\ln B_{n}$

$2 \cdot 3$ The lower bound for $\ln \left|g_{c}^{n}(c)\right|$

3 Proof of Proposition $2 \cdot 2$

4 Theorem 1.4 implies Theorem 1.2

References
\end{abstract}

\section{Introduction}

For every polynomial $f(x) \in \mathbb{Q}[x]$ and $\alpha \in \mathbb{Q}$ we put $f_{\alpha}(x):=f(x)+\alpha$. Therefore, $f_{\alpha}$ can be considered as a one-parameter family of polynomials. For every $u \in \mathbb{Q}$ we write

$$
\mathbb{S}_{f, u}:=\left\{c \in \mathbb{Q} \mid\left\{f_{c}^{n}(u)-u\right\}_{n \geqslant 1} \text { is infinite }\right\},
$$

where $f_{c}^{n}$ is the $n$-st iteration of $f_{c}$. In particular, if $u=0$, we put $\mathbb{S}_{f}:=\mathbb{S}_{f, 0}$.

We denote by $\operatorname{val}_{p}(-)$ the $p$-adic valuation of $\mathbb{Q}$ normalized by $\operatorname{val}_{p}(p)=1$. In keeping with the terminology of [5], for every polynomial $f(x) \in \mathbb{Q}[x], u \in \mathbb{Q}$ and $n \geqslant 1$ we say that $p$ is a primitive prime divisor of $f^{n}(u)-u$ if $\operatorname{val}_{p}\left(f^{n}(u)-u\right)>0$ and $\operatorname{val}_{p}\left(f^{k}(u)-u\right) \leqslant 0$ for all $1 \leqslant k<n$.

Definition 1-1. The Zsigmondy set of the sequence $\left\{f^{n}(u)-u\right\}_{n \geqslant 1}$ is defined by

$$
\mathcal{Z}(f, u):=\left\{n \geqslant 1 \mid f^{n}(u)-u \text { has no primitive prime divisor }\right\} .
$$

The primary application of bounds on the Zsigmondy set is towards understanding 
arboreal Galois representations associated to iteration of rational maps over number fields. It is first studied by Bang [1] and Zsigmondy [9]. Since then, there have been quite a few research papers on characterizing/bounding Zsigmondy sets of various sequences in various settings, e.g., Carmichael [2], Schinzel [8, Rice [7, Ingram-Silverman [5], Doerksen-Haensch [3, Gratton-Nguyen-Tucker [4, Krieger [6], etc.

In this work, we are interested in the size of the Zsigmondy set of a sequence obtained from the critical orbit of polynomials with rational coefficients of degree $d \geqslant 2$. We denote by $\Sigma$ the set of finite primes of $\mathbb{Z}$ and reserve $p$ for a prime number.

We first state our main theorem.

Theorem 1.2. For every polynomial $f \in \mathbb{Q}[x]$ of degree $d \geqslant 2$ with a critical point $u \in \mathbb{Q}$ there is a constant $\mathbf{M}_{f}>0$, depending only on $f$ (independent of $c \in \mathbb{Q}$ ), such that

$$
\# \mathcal{Z}\left(f_{c}, u\right) \leqslant \mathbf{M}_{f}
$$

for every $c \in \mathbb{S}_{f, u}$.

It is worth mentioning that Rice [7] was the first to prove the finiteness of $\mathcal{Z}(f, 0)$ for each individual polynomial $f(x) \neq x^{d}$. In [3], Doerksen-Haensch prove Theorem [1.2] for the case that $f(x)=x^{d}, u=0$ and $c \in \mathbb{Z}$, which is generalized by Krieger in [6] to every $c \in \mathbb{Q}$, see $[\mathbf{6}$, Theorem 1.1]. Our contribution is to prove Theorem 1.2 for general polynomials which are not necessary to be monic nor integer. As we consider polynomials $f$ that are more complicated than $x^{d}$, we did not aim to get the sharpest uniform bound $\mathbf{M}_{f}$.

Definition 1.3. A polynomial $g(x) \in \mathbb{Q}[x]$ is called $x^{2}$-divisible if it has degree $d \geqslant 2$ and is of the form

$$
g(x)=u_{d} x^{d}+\cdots+u_{2} x^{2} \in \mathbb{Q}[x] .
$$

At the last section we will prove that the following theorem implies Theorem 1.2,

Theorem 1.4. Given an $x^{2}$-divisible $g(x) \in \mathbb{Z}[x]$ of degree $d \geqslant 3$ there is a constant $\mathbf{M}_{g}>0$, depending only on $g$, such that

$$
\# \mathcal{Z}\left(g_{c}, 0\right) \leqslant \mathbf{M}_{g}
$$

for every $c \in \mathbb{S}_{g}$.

Note that one can give an explicit expression of the lower bound $\mathbf{M}_{g}$ when combining the decomposition of $\mathbb{S}_{g}$ in Proposition 2.2 with Propositions 3.3, 3.4, 3.7 and 3.8

This paper is inspired by Krieger's work in [6. We generalize her result from the special polynomial $f(x)=x^{d}$ to arbitrary polynomials in $\mathbb{Q}[x]$. We first address the difficulties on this generalization as follows.

The first difficulty is from dealing with the non-monic case, in which the denominator $f_{c}^{n}(c)$ is no longer always equal to $d^{n}$ 's power of the denominator of $c$. To conquer it, we introduce a factorization of an integer with respect to the leading term $u_{d}$ of $f$, see (2.9), which allows us to focus on the major factor of the denominator of $f_{c}^{n}(c)$.

The second difficulty is from the critical points of large multiplicities. Due to this reason, some arguments in [6 do not work for our case. For example, Krieger uses Mahler's theorem to control $\left|f_{c}^{n}(c)\right|$ by $\left|f_{c}^{n-1}(c)-f_{c}^{-1}(0)\right|$. However, this estimation might not be enough when $f_{c}^{n-1}(c)$ is very close to a critical point with large multiplicity. It forces us to control $\left|f_{c}^{n}(c)\right|$ in Proposition $2 \cdot 11$ by $\left|f_{c}^{n-N}(c)-f_{c}^{-N}(0)\right|$ for some relatively large $N>1$. 
Primitive prime divisors in the critical orbits of rational polynomials

\section{Acknowledgment}

The author would like to thank Tom Tucker and Shenhui Liu for their valuable discussions.

\section{Introduction of Proposition 2.2 and some estimates}

We split this section into two parts. In the first part, we introduce our main technical result Proposition $2 \cdot 2$ whose proof will be given in 83 , and prove that it implies Theorem 1.4. In the second part, we focus on estimating $\ln \left|A_{n}\right|$ which appears in Proposition $2 \cdot 2$,

Let us first set conventions and introduce some notations.

(1) We set $\mathbb{N}:=\{1,2, \ldots\}$ to be the set of natural numbers and for every $n \in \mathbb{N}$ we denote by $[n]$ the finite set $\{1,2, \ldots, n\}$.

(2) We denote by $\Sigma$ the set of finite primes of $\mathbb{Z}$ and reserve $p$ for a prime number. For every $n \in \mathbb{Z} \backslash\{0\}$ the sum $\sum_{p \mid n}$ and the product $\prod_{p \mid n}$ are taken over all its distinct prime factors whose number is denoted by $\omega(n)$.

We will always write an $x^{2}$-divisible $g(x) \in \mathbb{Z}[x]$ by $g(x)=u_{d} x^{d}+\cdots+u_{2} x^{2} \in \mathbb{Z}[x]$, and define its length by

$$
L_{g}:=1+\sum_{i=2}^{d-1}\left|u_{i}\right| /\left|u_{d}\right|
$$

For every $x^{2}$-divisible $g(x) \in \mathbb{Z}[x], c \in \mathbb{Q}$ and $n \geqslant 0$ we write the $(n+1)$-st iteration $g_{c}^{n+1}(0)$ as

$$
g_{c}^{n+1}(0)=g_{c}^{n}(c):=\frac{A_{n}}{B_{n}},
$$

where $B_{n}>0, A_{n}$ are coprime and both depend on $g$ and $c$. Clearly, we have $c=g_{c}(0)=$ $\frac{A_{0}}{B_{0}}$.

Definition 2-1. For an $x^{2}$-divisible $g(x) \in \mathbb{Z}[x]$ and a set $S$ in $\mathbb{S}_{g}$ we call that $g$ has rapidly increasing numerators on $S$ if there exists an integer $N>0$ such that for every $c \in S$ there is a finite set $J_{c}$ with $\# J_{c} \leqslant N$ such that for every $n \notin J_{c}$ we have

$$
\ln \left|A_{n}\right|>\sum_{p \mid n} \ln \left|A_{n / p}\right| \text {. }
$$

We now state our main proposition, which is followed by the proof of Theorem 1.4. Proposition 2.2 (Main Proposition). Every $x^{2}$-divisible $g(x) \in \mathbb{Z}[x]$ has rapidly increasing numerators on $\mathbb{S}_{g}$.

Proof of Theorem 1.4 in assuming proposition [2.2, By [6, Lemma 2.3 and Corollary 2.4], if $n \in \mathcal{Z}\left(g_{c}, 0\right)$, then $A_{n} \mid \prod_{p \mid n} A_{n / p}$ and hence

$$
\ln \left|A_{n}\right| \leqslant \sum_{p \mid n} \ln \left|A_{n / p}\right| .
$$

Together with Proposition 2.2, this finishes the proof.

The naive idea of proving Proposition $2 \cdot 2$ is to give a lower bound for $\ln \left|A_{n}\right|$ and an upper bound for $\prod_{p \mid n}\left|A_{n / p}\right|$ such that the lower bound is always greater than the upper bound when $n$ is large enough. Consider that

$$
\ln \left|A_{n}\right|=\ln B_{n}+\ln \left|g_{c}^{n}(c)\right| .
$$


It is sufficient for us to control $\ln B_{n}$ and $\ln \left|g_{c}^{n}(c)\right|$.

2.1. Upper bounds for $\ln B_{n}$ and $\ln \left|g_{c}^{n}(c)\right|$

Lemma 2-3. Given any $x^{2}$-divisible $g(x) \in \mathbb{Z}[x]$ and $c \in \mathbb{Q}$, for every $n \geqslant 0$ we have

(1) $\ln B_{n} \leqslant d^{n} \ln B_{0}$

(2) $\ln \left|g_{c}^{n}(c)\right| \leqslant d^{n} \ln \left(2\left|u_{d}\right| \max \left\{|c|, 4 L_{g}\right\}\right)$.

Proof. (1) Since $g_{c}^{n}(c)$ can be written as $A_{n}^{\prime} / B_{0}^{d^{n}}$ for some $A_{n}^{\prime} \in \mathbb{Z}$, we have $B_{n} \mid B_{0}^{d^{n}}$.

(2) It is enough to prove

$$
\left|g_{c}^{n}(c)\right| \leqslant\left(2\left|u_{d}\right|\right)^{\frac{d^{n}-1}{d-1}}\left(\max \left\{|c|, 4 L_{g}\right\}\right)^{d^{n}} \text { for all } n \geqslant 0 .
$$

For $n=0$, we have $\left|g_{c}^{0}(c)\right|=|c| \leqslant \max \left\{|c|, 4 L_{g}\right\}$.

Assume that the desired inequality holds for some $n \geqslant 0$ and temporarily denote its right side by $T_{n}$. Then we have

$$
\begin{aligned}
\left|g_{c}^{n+1}(c)\right| \leqslant\left|u_{d}\right|\left|g_{c}^{n}(c)\right|^{d}+ & \sum_{i=2}^{d-1}\left|u_{i}\right|\left|g_{c}^{n}(c)\right|^{i}+|c| \leqslant\left|u_{d}\right|\left(T_{n}+L_{g}\right) T_{n}^{d-1} \\
& \leqslant\left|u_{d}\right| \cdot 2 T_{n} \cdot T_{n}^{d-1}=\left(2\left|u_{d}\right|\right)^{\frac{d^{n+1}-1}{d-1}}\left(\max \left\{|c|, 4 L_{g}\right\}\right)^{d^{n+1}} .
\end{aligned}
$$

The proof follows by induction.

2.2. A lower bound for $\ln B_{n}$

Consider that

$$
\frac{A_{n+1}}{B_{n+1}}=g_{c}^{n+1}(c)=g_{c}\left(\frac{A_{n}}{B_{n}}\right)=\sum_{i=2}^{d} u_{i} \frac{A_{n}^{i}}{B_{n}^{i}}+\frac{A_{0}}{B_{0}} .
$$

Lemma 2.4. Given any $x^{2}$-divisible $g(x) \in \mathbb{Z}[x]$ and $c \in \mathbb{Q}$, for every $n \geqslant 0$ if $p \in I\left(B_{n}\right)$, then we have

(i) $p \in I\left(B_{n+1}\right)$ and

(ii) $\operatorname{val}_{p}\left(B_{n+1}\right)=d \operatorname{val}_{p}\left(B_{n}\right)-\operatorname{val}_{p}\left(u_{d}\right)$.

Proof. Note that for every $n \geqslant 0$ we have

$$
\begin{aligned}
\operatorname{val}_{p}\left(\frac{u_{d} A_{n}^{d}}{B_{n}^{d}}\right) & =\operatorname{val}_{p}\left(u_{d}\right)+d \operatorname{val}_{p}\left(A_{n}\right)-d \operatorname{val}_{p}\left(B_{n}\right), \\
\operatorname{val}_{p}\left(\frac{u_{i} A_{n}^{i}}{B_{n}^{i}}\right) & \geqslant(1-d) \operatorname{val}_{p}\left(B_{n}\right), \quad i=2, \ldots, d-1, \\
\operatorname{val}_{p}\left(\frac{A_{0}}{B_{0}}\right) & \geqslant-\operatorname{val}_{p}\left(B_{0}\right) .
\end{aligned}
$$

Therefore, if $p \in I\left(B_{n}\right)$, then we have $\operatorname{val}_{p}\left(A_{n}\right)=0$ and

$$
\operatorname{val}_{p}\left(\frac{u_{d} A_{n}^{d}}{B_{n}^{d}}\right)<(1-d) \operatorname{val}_{p}\left(B_{n}\right) \leqslant \operatorname{val}_{p}\left(\frac{u_{i} A_{n}^{i}}{B_{n}^{i}}\right)
$$

for every $2 \leqslant i \leqslant d-1$. Note that the term on the right hand side of this inequality does not exist for the case $d=2$.

We now prove this lemma by induction. 
Primitive prime divisors in the critical orbits of rational polynomials

For $n=0$ we have $\operatorname{val}_{p}\left(B_{0}\right)>\operatorname{val}_{p}\left(u_{d}\right)$. Combined with (2.3) and (2.7), this implies

$$
\operatorname{val}_{p}\left(\frac{A_{1}}{B_{1}}\right)=\operatorname{val}_{p}\left(\frac{u_{d} A_{0}^{d}}{B_{0}^{d}}\right)=\operatorname{val}_{p}\left(u_{d}\right)-d \operatorname{val}_{p}\left(B_{0}\right)<-\operatorname{val}_{p}\left(u_{d}\right)
$$

and hence $p \in I\left(B_{1}\right)$.

Now we assume that this lemma holds for every $0 \leqslant k \leqslant n$.

(1) If $p \notin I\left(B_{0}\right)$, we have

$$
\operatorname{val}_{p}\left(B_{0}\right) \leqslant \operatorname{val}_{p}\left(u_{d}\right)<\operatorname{val}_{p}\left(B_{n}\right) .
$$

Combined with (2.3) and (2.7), this implies

$$
\operatorname{val}_{p}\left(\frac{A_{n+1}}{B_{n+1}}\right)=\operatorname{val}_{p}\left(\frac{u_{d} A_{n}^{d}}{B_{n}^{d}}\right)=\operatorname{val}_{p}\left(u_{d}\right)-d \operatorname{val}_{p}\left(B_{n}\right)<-\operatorname{val}_{p}\left(u_{d}\right)
$$

and hence $p \in I\left(B_{n+1}\right)$.

(2) If $p \in I\left(B_{0}\right)$, then by induction, we have $p \in I\left(B_{n}\right)$ and

$$
\operatorname{val}_{p}\left(B_{n}\right)=d^{n} \operatorname{val}_{p}\left(B_{0}\right)-\operatorname{val}_{p}\left(u_{d}\right) \sum_{i=0}^{n-1} d^{i} \geqslant \operatorname{val}_{p}\left(B_{0}\right)
$$

Combining (2.3) with (2.7), we also obtain (2.8). This completes the induction.

For every $a \in \mathbb{Z}$ we denote

$$
I(a):=\left\{p \in \Sigma \mid \operatorname{val}_{p}(a)>\operatorname{val}_{p}\left(u_{d}\right)\right\},
$$

and put

$$
\widehat{a}:=\prod_{p \in I(a)} p^{\operatorname{val}_{p}(a)} .
$$

When $I(a)$ is empty, we put $\hat{a}:=1$. Note that we always have

$$
|a| \leqslant\left|u_{d} \widehat{a}\right| .
$$

Lemma 2.5. Given any $x^{2}$-divisible $g(x) \in \mathbb{Z}[x]$ of degree $d \geqslant 3$ and $c \in \mathbb{Q}$, for every $0 \leqslant n^{\prime} \leqslant n$ we have

$$
\ln B_{n} \geqslant \frac{d^{n-n^{\prime}}}{3} \ln \widehat{B_{n^{\prime}}}
$$

Proof. If $\widehat{B_{n^{\prime}}}=1$, it is trivial.

Now we assume that $\widehat{B_{n^{\prime}}} \geqslant 2$. It is enough to show that every prime $p \in I\left(B_{n^{\prime}}\right)$ satisfies

$$
\operatorname{val}_{p}\left(B_{n}\right) \geqslant \frac{d^{n-n^{\prime}}}{3} \operatorname{val}_{p}\left(B_{n^{\prime}}\right)
$$

Using Lemma 2.4 inductively, we have

$$
\operatorname{val}_{p}\left(B_{n}\right)=d^{n-n^{\prime}} \operatorname{val}_{p}\left(B_{n^{\prime}}\right)-\operatorname{val}_{p}\left(u_{d}\right) \sum_{i=0}^{n-n^{\prime}-1} d^{i} \geqslant\left(d^{n-n^{\prime}}-\sum_{i=0}^{n-n^{\prime}-1} d^{i}\right) \operatorname{val}_{p}\left(B_{n^{\prime}}\right) .
$$

From our assumption that $d \geqslant 3$, we have

$$
d^{n-n^{\prime}}-\sum_{i=0}^{n-n^{\prime}-1} d^{i} \geqslant d^{n-n^{\prime}}-2 d^{n-n^{\prime}-1} \geqslant \frac{1}{3} d^{n-n^{\prime}},
$$

which completes the proof. 
2.3. The lower bound for $\ln \left|g_{c}^{n}(c)\right|$

Lemma 2.6. Given any $x^{2}$-divisible $g(x) \in \mathbb{Z}[x]$ and $c \in \mathbb{Q}$, if $\left|g_{c}^{n^{\prime}}(c)\right| \geqslant \max \left\{4 L_{g},|c|\right\}$ for some $n^{\prime} \geqslant 0$, then for every $n \geqslant n^{\prime}$ we have

$$
\left|g_{c}^{n}(c)\right| \geqslant 2^{-\frac{d^{n-n^{\prime}}-1}{d-1}} \cdot\left|g_{c}^{n^{\prime}}(c)\right|^{d^{n-n^{\prime}}} .
$$

Clearly, in this case $c$ is in the basin of infinity for $g_{c}$.

Proof. The proof follows from induction. For $n=n^{\prime}$ this lemma is trivial.

Assume that this lemma holds for some $n \geqslant n^{\prime}$. Then we have

$$
\left|g_{c}^{n}(c)\right| \geqslant 2^{-\frac{d^{n-n^{\prime}}-1}{d-1}} \cdot\left|g_{c}^{n^{\prime}}(c)\right|^{d^{n-n^{\prime}}} \geqslant \max \left\{4 L_{g},|c|\right\} \cdot\left|4 L_{g} / 2\right|^{\frac{d^{n-n^{\prime}}-1}{d-1}} \geqslant \max \left\{4 L_{g},|c|\right\},
$$

and hence

$$
\begin{aligned}
\left|g_{c}^{n+1}(c)\right| \geqslant\left|u_{d}\right|\left|g_{c}^{n}(c)\right|^{d}-\sum_{i=2}^{d}\left|u_{i}\right|\left|g_{c}^{n}(c)\right|^{i}-|c| & \\
\geqslant\left|u_{d}\right|\left(\left|g_{c}^{n}(c)\right|^{d}-L_{g}\left|g_{c}^{n}(c)\right|^{d-1}\right) & =\left|g_{c}^{n}(c)\right|^{d-1}\left|u_{d}\right|\left(\left|g_{c}^{n}(c)\right|-L_{g}\right) \\
& \geqslant\left|g_{c}^{n}(c)\right|^{d} / 2 \geqslant 2^{-\frac{d^{n-n^{\prime}+1}-1}{d-1}} \cdot\left|g_{c}^{n^{\prime}}(c)\right|^{d^{n-n^{\prime}+1}} .
\end{aligned}
$$

Corollary 2.7. Given any $x^{2}$-divisible $g(x) \in \mathbb{Z}[x]$ and $c \in \mathbb{Q}$, if $\left|g_{c}^{n^{\prime}}(c)\right| \geqslant \max \left\{4 L_{g},|c|\right\}$ for some $n^{\prime} \geqslant 0$, then for every $n \geqslant n^{\prime}$ we have

$$
\ln \left|g_{c}^{n}(c)\right| \geqslant d^{n-n^{\prime}} \ln \left|g_{c}^{n^{\prime}}(c) / 2\right| .
$$

Proof. It follows directly from Lemma $2 \cdot 6$,

Given an algebraic number $\gamma \in \mathbb{C}$ of degree $\ell$ with conjugates $\gamma_{1}:=\gamma, \gamma_{2}, \ldots, \gamma_{\ell}$ over $\mathbb{Q}$, let $a_{0}$ be an integer such that the coefficients of the polynomial $g(X)=a_{0} \prod_{i=1}^{\ell}\left(X-\gamma_{i}\right)$ are integers of gcd 1, then we define the Mahler measure of $\gamma$ by

$$
M(\gamma):=\left|a_{0}\right| \prod_{i=1}^{\ell} \max \left(1,\left|\gamma_{i}\right|\right) .
$$

Notation 2.8. For every $r \geqslant 1$ and $\delta>0$ we put

$$
W(r, \delta):=2 \times 10^{7} \delta^{-4} \cdot \ln 4 r \cdot \ln \ln 4 r .
$$

Theorem 2.9 (10], Theorem 1). Let $0<\delta<1$. Then for every algebraic number $\gamma$ of degree $r \geqslant 1$, there are at most $W(r, \delta)$ solutions $a / b \in \mathbb{Q}$ to

$$
|a / b-\gamma|<M(a / b)^{-2-\delta}
$$

with $M(a / b) \geqslant \max \left\{4^{2 / \delta}, M(\gamma)\right\}$.

Theorem 2.9 implies the following result.

Corollary 2.10. Given any $x^{2}$-divisible $g(x) \in \mathbb{Z}[x]$, for every $L>0, N \in \mathbb{N}, c \in$ $[-L, L] \cap \mathbb{Q}$ and $\gamma \in \mathbb{C}$ such that $g_{c}^{N}(\gamma)=0$, there is an integer $D>0$, independent of $c$, such that

$$
|a / b-\gamma|<\frac{1}{(2 b D)^{3}}
$$

has at most $W\left(d^{N}, 1 / 10\right)$ rational solutions $a / b$ with $b \geqslant \max \left\{4^{20},\left(\left|u_{d}\right| B_{0} D\right)^{d^{N}}\right\}$. 
Primitive prime divisors in the critical orbits of rational polynomials

Proof. Let $\gamma_{c, 1}, \ldots, \gamma_{c, d^{N}}$ be the roots of $g_{c}^{N}(x)=0$ in $\mathbb{C}$ which are not necessary to be distinct. Since $g_{c}^{N}(x)$ is continuous as a function of $x$ and $c$, there exists an integer $D>1$ such that for every $c \in[-L, L] \cap \mathbb{Q}$ and $1 \leqslant i \leqslant d^{N}$ we have

$$
\left|\gamma_{c, i}\right|<D \text {. }
$$

Without loss of generality, we put $\gamma:=\gamma_{c, 1}$ and $h(x):=a_{0} \prod_{i=1}^{\ell}\left(x-\gamma_{c, i}\right)$ to be the minimal polynomial of $\gamma$ with integer coefficients of gcd 1 .

Since $B_{0}^{d^{N}} g_{c}^{N}(\gamma)=0$ and $B_{0}^{d^{N}} g_{c}^{N}(x)$ is a polynomial with integer coefficients, we have $h(x) \mid B_{0}^{d^{N}} g_{c}^{N}(x)$. Combined with Gauss's lemma, this implies

$$
a_{0} \mid\left(u_{d} B_{0}\right)^{d^{N}} \text {. }
$$

Combining (2.15) with (2.16), we have

$$
M(\gamma)<\left(\left|u_{d}\right| B_{0}\right)^{d^{N}} D^{\ell} .
$$

On the other hand, for every rational number $a / b$ in the lowest terms such that $|a / b| \leqslant$ $2 D$ we have

$$
b \leqslant M(a / b) \leqslant 2 b D .
$$

Note that Theorem 2.9] still holds when we do the following modifications.

(1) Restricting this theorem to a set of algebraic numbers and changing $M(\gamma)$ to a function of $\gamma$ which is larger than $M(\gamma)$ for every $\gamma$ in this set.

(2) Changing the right hand side of (2.13) to a function of $a / b$ which is less than $M(a / b)^{-2-\delta}$ for every rational number $a / b$.

(3) Changing the second $M(a / b)$ in Theorem 2.9 to a function of $a / b$ which is less than $M(a / b)$ for every rational number $a / b$.

Therefore, combined with (2.17) and (2.18), Theorem 2.9] implies that there are at most $W(\ell, \delta)$ rational solutions $a / b$ to

$$
|a / b-\gamma|<(2 b D)^{-2-\delta}
$$

such that $|a / b| \leqslant 2 D$ and $b \geqslant \max \left\{4^{2 / \delta},\left(\left|u_{d}\right| B_{0}\right)^{d^{N}} D^{\ell}\right\}$.

For rational number $a / b$ such that $|a / b| \geqslant 2 D$ we have

$$
|a / b-\gamma| \geqslant D>1>(2 b D)^{-2-\delta} .
$$

Together with (2.19), this shows that there at most $W(\ell, \delta)$ rational solutions $a / b \in \mathbb{Q}$ to

$$
|a / b-\gamma|<(2 b D)^{-2-\delta}
$$

with $b \geqslant \max \left\{4^{2 / \delta},\left(\left|u_{d}\right| B_{0}\right)^{d^{N}} D^{\ell}\right\}$.

Take $\delta:=1 / 10$. Combining $W(\ell, 1 / 10) \leqslant W\left(d^{N}, 1 / 10\right)$ with the modification(3) above, we can replace $\ell$ by $d^{N}$ and $-2-1 / 10$ by -3 , which completes the proof.

Now we consider $|c| \leqslant 4 L_{g}$. Recall that $\mathbb{S}_{g}$ is the set that contains all rational number $c$ such that $\left\{g_{c}^{n}(0)\right\}$ is infinite. By Corollary $2 \cdot 7$ with $n^{\prime}=0$, for every $c \in\left(-\infty,-4 L_{g}\right] \cup$ $\left[4 L_{g}, \infty\right)$ we have $\lim _{n \rightarrow \infty} \ln \left|g_{c}^{n}(c)\right|=\infty$ and hence $\mathbb{S}_{g} \supset\left(-\infty,-4 L_{g}\right] \cup\left[4 L_{g}, \infty\right)$.

We denote by $\mathbb{U}_{g}^{0}$ the finite subset of $\mathbb{S}_{g} \cap\left[-4 L_{g}, 4 L_{g}\right]$ consisting of all the rational numbers with denominator dividing $u_{d}$ and put $\mathbb{U}_{g}:=\left[-4 L_{g}, 4 L_{g}\right] \cap\left(\mathbb{S}_{g} \backslash \mathbb{U}_{g}^{0}\right)$. The following proposition aims at dealing the case $c \in \mathbb{U}_{g}$. It is worth noting that $\widehat{B_{0}} \geqslant 2$ for all $c \in \mathbb{U}_{g}$. 
Proposition 2-11. For an $x^{2}$-divisible $g(x) \in \mathbb{Z}[x]$ and a real number $\alpha \in\left[-4 L_{g}, 4 L_{g}\right]$ such that $g(x) \neq u_{d} x^{d}$ or $\alpha \neq 0$, there exists $0<\delta<L_{g}, C>0$ and an integer $N \geqslant 0$ such that for every $c \in(\alpha-\delta, \alpha+\delta) \cap \mathbb{S}_{g}$ if $\widehat{B_{n^{\prime}}} \geqslant 2$ for some $n^{\prime} \geqslant 0$, then there is a finite set $S_{c} \subset \mathbb{N}$ of bounded cardinality $N+n^{\prime}$ such that for every $n \notin S_{c}$ we have

$$
\ln \left|g_{c}^{n}(c)\right| \geqslant \min \left\{(-1+1 / d) \ln B_{n}+\ln C, \ln \delta\right\} .
$$

Proof. Let

$$
N_{0}:=\left\lceil\frac{2 \ln 3}{\ln (d /(d-1))}\right\rceil+1
$$

which satisfies

$$
9(d-1)^{N_{0}-1} \leqslant d^{N_{0}-1} .
$$

Let $\gamma_{1}, \ldots, \gamma_{r} \in \mathbb{C}$ be the distinct roots of $g_{\alpha}^{N_{0}}(x)=0$ of multiplicity $m_{1}, \ldots, m_{r}$, respectively. Choose an $0<\epsilon<1$ small enough such that for any two distinct $i, j \in[r]$ we have $\left|\gamma_{i}-\gamma_{j}\right|>3 \epsilon$.

By continuity of $g_{c}^{N}(x)$ as a function of $x$ and $c$, there exists $0<\delta<L_{g}$ such that for every $1 \leqslant i \leqslant r$ and $\alpha^{\prime}, \beta \in \mathbb{R}$ with $\left|\alpha^{\prime}-\alpha\right|<\delta$ and $|\beta|<\delta$ there are exactly $m_{i}$ roots of $g_{\alpha^{\prime}}^{N_{0}}(x)-\beta=0$ in the disk $O\left(\gamma_{i}, \epsilon\right) \subset \mathbb{C}$.

Now we consider an arbitrary $c \in(\alpha-\delta, \alpha+\delta) \cap \mathbb{S}_{g}$.

Let $\Gamma$ be the multiset consisting of all the roots of $g_{c}^{N_{0}}(x)=0$, i.e. two elements in $\Gamma$ could be the same. From the argument above, for every $n \geqslant N_{0}$ if $\left|g_{c}^{n}(c)\right|<\delta$, then there exists $1 \leqslant i_{0} \leqslant r$ such that $g_{c}^{n-N_{0}}(c) \in O\left(\gamma_{i_{0}}, \epsilon\right)$. We put

$$
\Gamma_{1}:=\Gamma \backslash O\left(\gamma_{i_{0}}, \epsilon\right) \quad \text { and } \quad \Gamma_{2}:=\Gamma \cap O\left(\gamma_{i_{0}}, \epsilon\right) .
$$

Note that we have

$$
\Gamma=\Gamma_{1} \cup \Gamma_{2}, \#(\Gamma)=d^{N_{0}} \text { and } \#\left(\Gamma_{2}\right)=m_{i_{0}} .
$$

Now we count the distance between $g_{c}^{n-N_{0}}(c)$ and the points in $\Gamma$.

For every $\xi \in \Gamma_{1}$, from our choice of $\epsilon$, we have

$$
\left|\xi-g_{c}^{n-N_{0}}(c)\right|>3 \epsilon-2 \epsilon=\epsilon .
$$

For every $\xi \in \Gamma_{2}$, by Corollary $2 \cdot 10$ with $L:=4 L_{g}$ and $N:=N_{0}$, there is an integer $D>0$, independent of $c$, such that

$$
|a / b-\xi|<\frac{1}{(2 b D)^{3}}
$$

has at most $W\left(d^{N_{0}}, 1 / 10\right)$ rational solutions $a / b$ with $b \geqslant \max \left\{4^{20},\left(\left|u_{d}\right| B_{0} D\right)^{d^{N_{0}}}\right\}$.

Put

$$
N_{1}:=\left\lceil\log _{d} 120\right\rceil+N_{0} \quad \text { and } \quad N_{2}:=\left\lceil 3 \log _{d} \log _{2}\left(u_{d}^{2} D+1\right)\right\rceil+2 N_{0} .
$$

Then for every $n \geqslant N_{1}$, by Lemma 2.5. we have

$$
\ln B_{n+n^{\prime}-N_{0}} \geqslant \frac{d^{n-N_{0}}}{3} \ln \widehat{B_{n^{\prime}}} \geqslant \frac{d^{n-N_{0}}}{3} \ln 2 \geqslant 20 \ln 4 .
$$

If $\widehat{B_{0}}=1$, by Lemma 2.5 (2.10) and the choice of $N_{2}$, for every $n \geqslant N_{2}$ we have

$$
\ln B_{n+n^{\prime}-N_{0}} \geqslant \frac{d^{n-N_{0}}}{3} \ln \widehat{B_{n^{\prime}}} \geqslant \frac{d^{n-N_{0}}}{3} \ln 2 \geqslant d^{N_{0}} \ln \left(u_{d}^{2} D\right) \geqslant d^{N_{0}} \ln \left(\left|u_{d}\right| B_{0} D\right) .
$$


Primitive prime divisors in the critical orbits of rational polynomials

If $\widehat{B_{0}} \geqslant 2$, by Lemma $2 \cdot 5$ and (2.10) again, for every $n \geqslant N_{2}$ we have

$$
\begin{aligned}
\ln B_{n-N_{0}} \geqslant \frac{d^{n-N_{0}}}{3} \ln \widehat{B_{0}} \geqslant\left(\frac{d^{n-N_{0}}}{3}-d^{N_{0}}\right) & \ln 2+d^{N_{0}} \ln \widehat{B_{0}} \\
& \geqslant d^{N_{0}} \ln \left(u_{d}^{2} \widehat{B_{0}} D\right) \geqslant d^{N_{0}} \ln \left(\left|u_{d}\right| B_{0} D\right) .
\end{aligned}
$$

Therefore, there are most $W\left(d^{N_{0}}, 1 / 10\right)$ many integers $n \geqslant \max \left\{N_{1}+n^{\prime}, N_{2}+n^{\prime}\right\}$ such that $g_{c}^{n-N_{0}}(c)$ is a rational solution to (2.24). Combined with

$$
\left|g_{c}^{n}(c)\right|=\left|g_{c}^{N_{0}}\left(g_{c}^{n-N_{0}}(c)\right)\right|=\prod_{\xi \in \Gamma}\left|g_{c}^{n-N_{0}}(c)-\xi\right|=\prod_{\xi \in \Gamma_{1}}\left|g_{c}^{n-N_{0}}(c)-\xi\right| \cdot \prod_{\xi \in \Gamma_{2}}\left|g_{c}^{n-N_{0}}(c)-\xi\right|,
$$

this implies that for all but at most $\# \Gamma_{2} \cdot W\left(d^{N_{0}}, 1 / 10\right)$ many $n \geqslant \max \left\{N_{1}+n^{\prime}, N_{2}+n^{\prime}\right\}$ we have

$$
\ln \left|g_{c}^{n}(c)\right| \geqslant \# \Gamma_{1} \cdot \ln \epsilon-3 \cdot \# \Gamma_{2} \ln \left(2 D B_{n-N_{0}}\right) .
$$

Combining (2.22) and our assumption $0<\epsilon<1$, we have

$$
\# \Gamma_{1} \cdot \ln \epsilon-3 \cdot \# \Gamma_{2} \ln \left(2 D B_{n-N_{0}}\right) \geqslant d^{N_{0}} \ln \left(\frac{\epsilon}{8 D^{3}}\right)-3 \# \Gamma_{2} \ln B_{n-N_{0}} .
$$

From our assumption that $g(x) \neq u_{d} x^{d}$ or $\alpha \neq 0$, we have

$$
\# \Gamma_{2}=m_{i_{0}} \leqslant(d-1)^{N_{0}} .
$$

Therefore, the previous statement implies that for all but at most $(d-1)^{N_{0}} W\left(d^{N_{0}}, 1 / 10\right)$ many $n \geqslant \max \left\{N_{1}+n^{\prime}, N_{2}+n^{\prime}\right\}$ we have

$$
\ln \left|g_{c}^{n}(c)\right| \geqslant d^{N_{0}} \ln \left(\frac{\epsilon}{8 D^{3}}\right)-3(d-1)^{N_{0}} \ln B_{n-N_{0}} .
$$

By (2.10) and (2.21), we have

$$
3(d-1)^{N_{0}} \ln B_{n-N_{0}} \leqslant \frac{d^{N_{0}-1}(d-1)}{3} \ln B_{n-N_{0}} \leqslant \frac{d^{N_{0}-1}(d-1)}{3} \ln \left(\left|u_{d}\right| \widehat{B_{n-N_{0}}}\right) .
$$

By Lemma 2.5, we obtain

$$
\begin{aligned}
& \frac{d^{N_{0}-1}(d-1)}{3} \ln \left(\left|u_{d}\right| \widehat{B_{n-N_{0}}}\right) \leqslant \frac{d^{N_{0}-1}(d-1)}{3} \ln \left|u_{d}\right|+\frac{d-1}{d} \ln B_{n} \\
& \quad \leqslant d^{N_{0}} \ln \left|u_{d}\right|+\frac{d-1}{d} \ln B_{n}
\end{aligned}
$$

The two inequality above implies that

$$
\text { Right Hand Side of (2.26) } \geqslant d^{N_{0}} \ln \left(\frac{\epsilon}{8\left|u_{d}\right| D^{3}}\right)+(-1+1 / d) \ln B_{n},
$$

which completes the proof.

\section{Proof of Proposition 2.2}

The basic idea of proving Proposition $2 \cdot 2$ is to show that for each $x^{2}$-divisible $g(x) \in$ $\mathbb{Z}[x]$ there exists a finite cover of $\mathbb{S}_{g}$ as follows:

(1) $\left(-\infty,-4 L_{g}\right] \cup\left[4 L_{g}, \infty\right)$;

(2) $\left(\alpha-\delta_{g, \alpha}, \alpha+\delta_{g, \alpha}\right) \cap \mathbb{U}_{g}$ for finitely many $\alpha$ in $\left[-4 L_{g}, 4 L_{g}\right]$ with $0<\delta_{g, \alpha}<L_{g}$;

(3) the finite set $\mathbb{U}_{g}^{0}$, 
such that $g$ has rapidly increasing numerators on every set in this cover.

Recall that for every $n \in \mathbb{N}$ we denote by $\omega(n)$ the number of its distinct prime divisors. For convenience, we put $s_{d}(n):=\sum_{p \mid n} d^{\frac{n}{p}}$. Then we have the following estimation.

Lemma 3.1. For every $d \geqslant 2$ and every $n \geqslant 30$, we have $s_{d}(n) \leqslant d^{\frac{3 n}{5}}$.

Proof. For every integer $n \geqslant 2$ we have $n \geqslant 2^{\omega(n)}$ and hence

$$
\omega(n) \leqslant \log _{2} n .
$$

Since for every prime divisor $p$ of $n$ we have $n / p \leqslant n / 2$. Combined with (3.1), we have

$$
s_{d}(n) \leqslant d^{\frac{n}{2}} \omega(n) \leqslant d^{\frac{n}{2}} \log _{2} n .
$$

On the other hand, for every $n \geqslant 30$ we have

$$
\log _{2} n<5<2^{3} \leqslant d^{\frac{n}{10}} .
$$

Together with (3.2), this finishes the proof.

Lemma 3.2. Given any $x^{2}$-divisible $g(x) \in \mathbb{Z}[x]$ and $c \in \mathbb{Q}$, for every $n \geqslant 30$ we have

$$
\sum_{p \mid n} \ln \left|A_{n / p}\right| \leqslant d^{\frac{3 n}{5}} \ln \left(2\left|u_{d}\right|^{2} \widehat{B_{0}} \max \left\{|c|, 4 L_{g}\right\}\right) .
$$

Proof. By Lemma 2.3, for every $n \geqslant 0$ we have

$$
\sum_{p \mid n} \ln \left|A_{n / p}\right| \leqslant \sum_{p \mid n} d^{n / p} \ln \left(2\left|u_{d}\right| B_{0} \max \left\{|c|, 4 L_{g}\right\}\right) .
$$

Together with Lemma $3 \cdot 1$ and (2.10), this finishes the proof.

Proposition 3.3. Every $x^{2}$-divisible $g(x) \in \mathbb{Z}[x]$ of degree $d \geqslant 3$ has rapidly increasing numerators on $\left(-\infty,-4 L_{g}\right] \cup\left[4 L_{g}, \infty\right)$.

Proof. Let $c$ be an arbitrary rational number in $\left(-\infty,-4 L_{g}\right] \cup\left[4 L_{g}, \infty\right)$.

By Lemma 2.5. Corollary 2.7 with $n^{\prime}=0$ and $|c| \geqslant 4 L_{g} \geqslant 4$, for every $n \geqslant 0$ we have

$$
\ln \left|A_{n}\right| \geqslant d^{n} \ln |c / 2|+\frac{d^{n}}{3} \ln \widehat{B_{0}} \geqslant \frac{d^{n}}{3} \ln \left|c \widehat{B_{0}}\right| .
$$

Combined with Lemma $3 \cdot 2$ and $|c| \geqslant 4 L_{g}$, this implies that for every $n \geqslant 30$ we have

$$
\ln \left|A_{n}\right|-\sum_{p \mid n} \ln \left|A_{n / p}\right| \geqslant-d^{\frac{3 n}{5}} \ln \left(2\left|u_{d}\right|^{2}\right)+\left(d^{n} / 3-d^{\frac{3 n}{5}}\right) \ln \left|c \widehat{B_{0}}\right| .
$$

Therefore, there exists an integer $N>\max \left\{\frac{5}{2} \log _{d} 3,30\right\}$, which only depends on $g$, such that for every $n \geqslant N$ and every rational number $c \in\left(-\infty,-4 L_{g}\right] \cup\left[4 L_{g}, \infty\right)$ we have

$$
\ln \left|A_{n}\right|>\sum_{p \mid n} \ln \left|A_{n / p}\right| .
$$

Thus we prove this proposition.

We next prove the following.

Proposition 3.4. Every polynomial $g(x)=u_{d} x^{d} \in \mathbb{Z}[x]$ of degree $d \geqslant 3$ has rapidly increasing numerators on $\left(-\frac{1}{\left|4 u_{d}\right|}, \frac{1}{\left|4 u_{d}\right|}\right) \cap \mathbb{S}_{g}$.

Since $0 \notin \mathbb{S}_{g}$, it is sufficient to show the following two lemmas. 
Primitive prime divisors in the critical orbits of rational polynomials 11

Lemma 3.5. Every polynomial $g(x)=u_{d} x^{d} \in \mathbb{Z}[x]$ of degree $d \geqslant 3$ has rapidly increasing numerators on $\left(0, \frac{1}{4\left|u_{d}\right|}\right) \cap \mathbb{S}_{g}$.

Proof. For every $c \in\left(0, \frac{1}{4\left|u_{d}\right|}\right) \cap \mathbb{S}_{g}$ and $n \geqslant 0$ we have

$$
|c| \leqslant\left|g_{c}^{n}(c)\right| \leqslant\left(\left|u_{d}\right|+1\right)^{\frac{d^{n}-1}{d-1}}|c| \leqslant\left(\left|u_{d}\right|+1\right)^{d^{n}}|c|
$$

and $\widehat{B_{0}} \geqslant 2$.

Combining Lemmas 2.3(1), 2.5 for $n^{\prime}=0$ with (3.4), for every $n \geqslant 0$ we have

$$
\ln \left|A_{n}\right|-\sum_{p \mid n} \ln \left|A_{n / p}\right| \geqslant(1-\omega(n)) \ln |c|+\frac{d^{n}}{3} \ln \widehat{B_{0}}-s_{d}(n) \ln \left(\left(\left|u_{d}\right|+1\right) B_{0}\right) .
$$

Together with (2.10) and lemma 3.1 this implies that for every $n \geqslant 30$ we have

$$
\ln \left|A_{n}\right|-\sum_{p \mid n} \ln \left|A_{n / p}\right| \geqslant-d^{\frac{3 n}{5}} \ln \left(\left(\left|u_{d}\right|+1\right)\left|u_{d}\right|\right)+\left(\frac{d^{n}}{3}-d^{\frac{3 n}{5}}\right) \ln \widehat{B_{0}} .
$$

From $\widehat{B_{0}} \geqslant 2$, there exists an integer $N \geqslant 30$ such that for every $n \geqslant N$ we have

$$
\ln \left|A_{n}\right|-\sum_{p \mid n} \ln \left|A_{n / p}\right|>0
$$

which completes the proof.

Lemma 3.6. Every polynomial $g(x)=u_{d} x^{d} \in \mathbb{Z}[x]$ of degree $d \geqslant 3$ has rapidly increasing numerators on $\left(-\frac{1}{4\left|u_{d}\right|}, 0\right) \cap \mathbb{S}_{g}$.

Proof. Note that when $d$ is odd, we may replace $c$ with $-c$ and the forward orbit of 0 will be unchanged, modulo sign. By Lemma 3.5 , we immediately prove this case.

Therefore, it is sufficient to study the case that $d$ is even. We first show that for every $c \in\left(-\frac{1}{4\left|u_{d}\right|}, 0\right)$ and every $n \geqslant 0$ we have

$$
|c|\left(1-\left|u_{d}\right||c|^{d-1}\right) \leqslant\left|g_{c}^{n}(c)\right| \leqslant|c| .
$$

For $n=0$, we have $\left|g_{c}^{0}(c)\right|=|c|$. Assume that (3.5) holds for some $n \geqslant 0$. Since $g_{c}(x)$ is negative and decreasing on $\left(-\frac{1}{4\left|u_{d}\right|}, 0\right)$, we have

$$
|c|\left(1-\left|u_{d}\right||c|^{d-1}\right)=\left|g_{c}(c)\right| \leqslant\left|g_{c}\left(g_{c}^{n}(c)\right)\right| \leqslant|f(0)|=|c|,
$$

which proves (3.5) by induction.

Combining Lemmas 2.3 (1), 2.5 with (3.5), we have

$$
\begin{array}{r}
\ln \left|A_{n}\right|-\sum_{p \mid n} \ln \left|A_{n / p}\right| \geqslant \ln \left(|c| \cdot\left(1-\left|u_{d}\right||c|^{d-1}\right)\right)+\frac{d^{n}}{3} \ln \widehat{B_{0}}-s_{d}(n) \ln B_{0}-\omega(n) \ln |c| \\
\geqslant \ln \left(1-\left|u_{d}\right||c|^{d-1}\right)+\frac{d^{n}}{3} \ln \widehat{B_{0}}-s_{d}(n) \ln B_{0} .
\end{array}
$$

Together with (2.10), Lemma $3 \cdot 1$ and $|c|<\frac{1}{4\left|u_{d}\right|}$, this implies that for every $n \geqslant 30$ we have

$$
\ln \left|A_{n}\right|-\sum_{p \mid n} \ln \left|A_{n / p}\right| \geqslant \ln (3 / 4)+d^{\frac{3 n}{5}} \ln \left|u_{d}\right|+\left(\frac{d^{n}}{3}-d^{\frac{3 n}{5}}\right) \ln \widehat{B_{0}} .
$$


On the other hand, for every $c \in\left(-\frac{1}{4\left|u_{d}\right|}, 0\right)$, we have $\widehat{B_{0}} \geqslant 2$. Combined with (3.6), this proves that there exists an integer $N \geqslant 30$ such that for every $n \geqslant N$ we have

$$
\ln \left|A_{n}\right|-\sum_{p \mid n} \ln \left|A_{n / p}\right|>0
$$

which completes the proof.

Proposition 3.7. Given any $x^{2}$-divisible $g(x) \in \mathbb{Z}[x]$ of degree $d \geqslant 3$ and $\alpha \in\left[-4 L_{g}, 4 L_{g}\right]$ such that $g(x) \neq u_{d} x^{d}$ or $\alpha \neq 0$, there is an $0<\delta<L_{g}$ such that $g$ has rapidly increasing numerators on $c \in(\alpha-\delta, \alpha+\delta) \cap \mathbb{U}_{g}$.

Proof. Note that for every $c \in \mathbb{U}_{g}$ we have $\widehat{B_{0}} \geqslant 2$. By Proposition $2 \cdot 11$ with $n^{\prime}=0$, there is a 3-tuple $0<\delta<L_{g}, C>0$ and $N_{1}>0$ such that for every $c \in(\alpha-\delta, \alpha+\delta) \cap \mathbb{U}_{g}$ there is a finite set $S_{c} \subset \mathbb{N}$ of bounded cardinality $N_{1}$ such that for every $n \notin S_{c}$ we have

$$
\ln \left|g_{c}^{n}(c)\right| \geqslant \min \left\{(-1+1 / d) \ln B_{n}+\ln C, \ln \delta\right\},
$$

and therefore

$$
\ln \left|A_{n}\right| \geqslant \min \left\{\frac{1}{d} \ln B_{n}+\ln C, \ln B_{n}+\ln \delta\right\} .
$$

On the other hand, by Lemma 2.5, we have

$$
\begin{gathered}
\ln \left(B_{n}\right)+\ln \delta-d^{\frac{3 n}{5}} \ln \left|2 c u_{d}^{2} \widehat{B_{0}}\right| \geqslant \ln \delta+\left(\frac{d^{n}}{3}-d^{\frac{3 n}{5}}\right) \ln \widehat{B_{0}}-d^{\frac{3 n}{5}} \ln \left|2 c u_{d}^{2}\right|, \\
\frac{1}{d} \ln \left(B_{n}\right)+\ln C-d^{\frac{3 n}{5}} \ln \left|2 c u_{d}^{2} \widehat{B_{0}}\right| \geqslant \ln C+\left(\frac{d^{n-1}}{3}-d^{\frac{3 n}{5}}\right) \ln \widehat{B_{0}}-d^{\frac{3 n}{5}} \ln \left|2 c u_{d}^{2}\right| .
\end{gathered}
$$

Combined with Lemma 3.2. (3.7) and $|c| \leqslant 5 L_{g}$, this implies that there exists an integer $N_{2} \geqslant 30$ such that for every rational number $c \in(\alpha-\delta, \alpha+\delta)$ and $n \in\left\{N_{2}, N_{2}+1, \ldots,\right\} \backslash S_{c}$ we have

$$
\ln \left|A_{n}\right|>\sum_{p \mid n} \ln \left|A_{n / p}\right| .
$$

Taking $N:=N_{1}+N_{2}$, we prove this proposition.

Now we turn our attention to the finite set $\mathbb{U}_{g}^{0}$.

Proposition 3.8. Every $x^{2}$-divisible $g(x) \in \mathbb{Z}[x]$ of degree $d \geqslant 3$ has rapidly increasing numerators on the finite set $\mathbb{U}_{g}^{0}$.

Proof. It is sufficient to show that for each individual rational number in $\mathbb{U}_{g}^{0}$ there are finite many $n \in \mathbb{N}$ satisfying (2.1).

Let $c$ be an arbitrary rational number in $\mathbb{U}_{g}^{0}$. We first show that there must exist an integer $n^{\prime}$ such that either $\left|g_{c}^{n^{\prime}}(c)\right|>4 L_{g}$ or $\widehat{B_{n^{\prime}}} \geqslant 2$. Suppose that for every $n \geqslant 0$ we have $\widehat{B_{n}}=1$, i.e., $B_{n} \mid u_{d}$. Since $c \in \mathbb{S}_{g}$ and there are only finitely many integers in $\left[-4 L_{g}, 4 L_{g}\right]$ with denominator dividing $u_{d}$, we know that there must exist an $n^{\prime} \geqslant 0$ such that $\left|g_{c}^{n^{\prime}}(c)\right|>4 L_{g}$.

(1) When $|c| \leqslant 4 L_{g}, \widehat{B_{0}}=1$ and there exists an integer $n^{\prime} \geqslant 1$ such that $\left|g_{c}^{n^{\prime}}(c)\right|>4 L_{g}$. Combining these conditions with Corollary $2 \cdot 7$ and Lemma $3 \cdot 2$, for every $n \geqslant \max \left\{30, n^{\prime}\right\}$ we have

$$
\ln \left|A_{n}\right|-\sum_{p \mid n} \ln \left|A_{n / p}\right| \geqslant-d^{\frac{3 n}{5}} \ln \left(8 L_{g}\left|u_{d}\right|^{2}\right)+d^{n-n^{\prime}}\left|g_{c}^{n^{\prime}}(c) / 2\right|
$$


Primitive prime divisors in the critical orbits of rational polynomials 13

Clearly, there exists an integer $N>\max \left\{30, n^{\prime}\right\}$ such that for every $n \geqslant N$ we have

$$
\ln \left|A_{n}\right|>\sum_{p \mid n} \ln \left|A_{n / p}\right| \text {. }
$$

(2) When $|c| \leqslant 4 L_{g}, \widehat{B_{0}}=1$ and there is an integer $n^{\prime} \geqslant 1$ such that $\widehat{B_{n^{\prime}}} \geqslant 2$. Similar to Proposition 3.7, we combine Lemma 2.5 with Proposition 2.11, and obtain a finite set $S_{c} \subset \mathbb{N}$, an integer $N, \delta_{c}>0$ and $C_{c}>0$ such that for every $n \geqslant n^{\prime}$ if $n \notin S_{c}$, then

$$
\ln \left|g_{c}^{n}(c)\right| \geqslant \min \left\{(-1+1 / d) \ln B_{n}+\ln C_{c}, \ln \delta_{c}\right\},
$$

and therefore

$$
\ln \left|A_{n}\right| \geqslant \min \left\{\frac{1}{d} \ln B_{n}+\ln C_{c}, \ln B_{n}+\ln \delta_{c}\right\} .
$$

On the other hand, by Lemma $2 \cdot 5$, we have

$$
\begin{gathered}
\ln B_{n}+\ln \delta_{c}-d^{\frac{3 n}{5}} \ln \left|2 c u_{d}^{2} \widehat{B_{0}}\right| \geqslant \ln \delta_{c}+\frac{d^{n-n^{\prime}}}{3} \ln \widehat{B_{n^{\prime}}}-d^{\frac{3 n}{5}} \ln \left|2 c u_{d}^{2}\right|, \\
\frac{1}{d} \ln B_{n}+\ln C_{c}-d^{\frac{3 n}{5}} \ln \left|2 c u_{d}^{2} \widehat{B_{0}}\right| \geqslant \ln C_{c}+\frac{d^{n-n^{\prime}-1}}{3} \ln \widehat{B_{n^{\prime}}}-d^{\frac{3 n}{5}} \ln \left|2 c u_{d}^{2}\right| .
\end{gathered}
$$

Combined with Lemma 3.2, (3.8) and $|c| \leqslant 4 L_{g}$, this implies that there exists an integer $N_{1}>\max \left\{n^{\prime}, 30\right\}$ such that for every $n \in\left\{N_{1}, N_{1}+1, \ldots\right\} \backslash S_{c}$ we have

$$
\ln \left|A_{n}\right|>\sum_{p \mid n} \ln \left|A_{n / p}\right| .
$$

Put $N:=\# S_{c}+N_{1}$. Then we complete the proof.

Proof of Proposition [2.2. By Proposition 3.7, for every $g(x) \neq u_{d} x^{d}$ or $\alpha \neq 0$ and every real number $\alpha \in\left[-4 L_{g}, 4 L_{g}\right]$, there is an $0<\delta_{g, \alpha}<L_{g}$ such that $g$ has rapidly increasing numerators on $c \in\left(\alpha-\delta_{g, \alpha}, \alpha+\delta_{g, \alpha}\right) \cap \mathbb{U}_{g}$.

For $g(x)=u_{d} x^{d}$ and $\alpha=0$, if we put $\delta_{g, \alpha}:=\frac{1}{4\left|u_{d}\right|}$, then we proved in Proposition $3 \cdot 4$ that $g$ has rapidly increasing numerators on $c \in\left(-\delta_{g, \alpha}, \delta_{g, \alpha}\right) \cap \mathbb{U}_{g}$.

Now for every $x^{2}$-divisible $g(x) \in \mathbb{Z}[x]$ we obtain a cover of $\mathbb{S}_{g}$ as

$$
\left(-\infty,-4 L_{g}\right] \cup\left[4 L_{g}, \infty\right) \cup \mathbb{U}_{g}^{0} \cup \bigcup_{\alpha \in\left[-4 L_{g}, 4 L_{g}\right]}\left(\left(\alpha-\delta_{g, \alpha}, \alpha+\delta_{g, \alpha}\right) \cap \mathbb{U}_{g}\right) .
$$

Note that

$$
\bigcup_{\alpha \in\left[-4 L_{g}, 4 L_{g}\right]}\left(\alpha-\delta_{g, \alpha}, \alpha+\delta_{g, \alpha}\right)
$$

is an open cover of the closed interval $\left[-4 L_{g}, 4 L_{g}\right]$, which has a finite cover. We use the center $\alpha$ to represent the interval $\left(\alpha-\delta_{g, \alpha}, \alpha+\delta_{g, \alpha}\right)$ in this finite cover, and put $T$ to be the index set of $\alpha$.

Therefore, we obtain a finite cover of $\mathbb{S}_{g}$ as follows:

$$
\left(-\infty,-4 L_{g}\right] \cup\left[4 L_{g}, \infty\right) \cup \mathbb{U}_{g}^{0} \cup \bigcup_{\alpha \in T}\left(\left(\alpha-\delta_{g, \alpha}, \alpha+\delta_{g, \alpha}\right) \cap \mathbb{U}_{g}\right) .
$$

By Propositions $3 \cdot 3,3 \cdot 4,3 \cdot 7$ and $3 \cdot 8$, we know that $g$ has rapidly increasing numerators on each set in this cover. It implies that $g$ also has rapidly increasing numerators on $\mathbb{S}_{g}$, which completes the proof. 


\section{Theorem 1.4 implies Theorem 1.2}

At the end of this section, we will prove that Theorem 1.4 implies the following proposition which leads to Theorem $1 \cdot 2$.

Proposition 4.1. For every $x^{2}$-divisible polynomial $g(x) \in \mathbb{Q}[x]$ there is a constant $\mathbf{M}_{g}>0$, depending only on $g$, such that

$$
\# \mathcal{Z}\left(g_{c}, 0\right) \leqslant \mathbf{M}_{g}
$$

for every $c \in \mathbb{S}_{g}$.

For every $n \in \mathbb{Z} \backslash\{0\}$, we denote by $\omega(n)$ the number of distinct prime factors of $n$.

Lemma 4.2. Let $g(x) \in \mathbb{Q}[x]$ be $x^{2}$-divisible and $a \in \mathbb{Z} \backslash\{0\}$. Then with $h(x):=\frac{1}{a} g(a x)$ we have

$$
\left|\# \mathcal{Z}\left(g_{c}, 0\right)-\# \mathcal{Z}\left(h_{c / a}, 0\right)\right| \leqslant \omega(a) \quad \text { for every } c \in \mathbb{Q} .
$$

Proof. Consider that

$$
g_{c}^{n}(a x)=u h_{c / a}^{n}(x) \quad \text { and } \quad g_{c}^{n}(0)=a h_{c / a}^{n}(0) .
$$

For every $p \nmid u$ we have $p$ is a primitive prime divisor of $g_{c}^{n}(0)$ if and only if it is a primitive prime divisor of $h_{c / a}^{n}(0)$. Therefore, the difference between $\# \mathcal{Z}\left(g_{c}, 0\right)$ and $\# \mathcal{Z}\left(h_{c / a}, 0\right)$ can not exceed the number of prime factors of $a$, which completes the proof.

Lemma 4.3. For any $x^{2}$-divisible $g(x) \in \mathbb{Q}[x]$ and $t \in \mathbb{Q} \backslash\{0\}$, Proposition $4 \cdot 1$ holds for $g$ if and only if it holds for $\frac{1}{t} g(t x)$.

Proof. Let $t=\frac{a}{b}$ be an arbitrary rational number. Put

$$
h(x):=\frac{1}{t} g(t x) \quad \text { and } \quad h_{1}(x):=\frac{1}{a} g(a x) .
$$

Note that $h_{1}(x)=\frac{1}{b} h(b x)$.

By Lemma $4 \cdot 2$ for every $c \in \mathbb{Q}$ we have

$$
\begin{aligned}
\left|\# \mathcal{Z}\left(g_{c}, 0\right)-\# \mathcal{Z}\left(\left(h_{1}\right)_{c / a}, 0\right)\right| & \leqslant \omega(a), \\
\left|\# \mathcal{Z}\left(h_{c / t}, 0\right)-\# \mathcal{Z}\left(\left(h_{1}\right)_{c / a}, 0\right)\right| & \leqslant \omega(b),
\end{aligned}
$$

which implies

$$
\left|\# \mathcal{Z}\left(g_{c}, 0\right)-\# \mathcal{Z}\left(h_{c / t}, 0\right)\right| \leqslant \omega(a)+\omega(b) .
$$

Since $\omega(a)$ and $\omega(b)$ are both independent of $c$, we complete the proof.

Proposition 4.4. Theorem 1.4 implies Theorem 1.2.

Proof. Step I: We first prove that Theorem 1.4 implies Proposition 4.1

Given any $x^{2}$-divisible polynomial $g(x) \in \mathbb{Q}[x]$ of degree $d \geqslant 2$, if $d=2$, then we can find $t \in \mathbb{Q}$ such that $\frac{1}{t} g(t x)=x^{2}$. Combining Lemma 4.3 with [6. Theorem 1.1], we prove Proposition 4.1 for this case.

If $d \geqslant 3$, there is $t \in \mathbb{Z}$ such that $\frac{1}{t} g(t x) \in \mathbb{Z}[x]$. Combined with Lemma 4.3, this proves Proposition $4 \cdot 1$ for this case.

Step II: Now we prove that Proposition $4 \cdot 1$ implies Theorem 1.2 .

For every polynomial $g(x) \in \mathbb{Q}[x]$ with a critical point $u \in \mathbb{Q}$, if we put $f(x):=$ $g(x+u)-u$, then we know that 0 is a critical point of $f$ and for every $c \in \mathbb{Q}$ we have $f_{c}^{n}(0)=g_{c}^{n}(u)-u$ and hence

$$
\mathcal{Z}\left(g_{c}, u\right)=\mathcal{Z}\left(f_{c}, 0\right)
$$


Primitive prime divisors in the critical orbits of rational polynomials 15

This completes the proof.

\section{REFERENCES}

[1] A. S. BAng. Talteoretiske undersøgelser. Tidsskrift Mat. (5) 4 (1886), 70-80; 130-137.

[2] R. D. Carmichael. On the numerical factors of the arithmetic forms $\alpha^{n} \pm \beta^{n}$. Ann. of Math. (1/4) 15 (1913), 49-70.

[3] K. Doerksen and A. Haensch. Primitive prime divisors in zero orbits of polynomials. Integers (3) 12 (2011), 465-473.

[4] C. Gratton, K. Nguyen, and T. J. Tucker. ABC implies primitive prime divisors in arithmetic dynamic. Bull. Lond. Math. Soc. (6) 45 (2013), 1194-1208.

[5] P. Ingram and J. Silverman. Primitive divisors in arithmetic dynamics. Math. Proc. Cambridge Philos. Soc. (2) 146 (2009), 289-302.

[6] H. Krieger. Primitive prime divisors in the critical orbit of $z^{d}+c$. Int. Math. Res. Not. IMRN 23 (2013), 5498-5525.

[7] B. RicE. Primitive prime divisors in polynomial arithmetic dynamics. Integers (1) 7 (2007), A26, 1-16.

[8] A. Schinzel. Primitive divisors of the expression $a^{n}-b^{n}$ in algebraic number fields. $J$. Reine Angew. Math. 268/269 (1974), 27-33.

[9] K. Zsigmondy. Zur Theorie der Potenzreste. Monatsh. Math. Phys. (1) 3 (1892), 265-284.

[10] J. H. EvERTSE. The number of algebraic numbers of given degree approximating a given algebraic number. Analytic Number Theory, Y. Motohashi (ed.), London Math. Soc. Lecture Notes Ser. 247, Cambridge Univ. Press (1998), 53-83. 